\title{
IV. On the mechanism of electrical conduction.-Part I. Conduction in metals
}

\section{Charles V. Burton D.Sc.}

To cite this article: Charles V. Burton D.Sc. (1894) IV. On the mechanism of electrical conduction.-Part I. Conduction in metals, Philosophical Magazine Series 5, 38:230, 55-70, DOI: $10.1080 / 14786449408620601$

To link to this article: http://dx.doi.org/10.1080/14786449408620601

曲 Published online: 08 May 2009.

Submit your article to this journal $\sqsubset x$

Џ Article views: 2

Q View related articles $\asymp$ 
the less viscous metals and increases with the period in other metals. According to the above theory this is due to the fact that a given state of stress is continued longer, so that the molecular configurations have more opportunity to break down.

If the theory were pushed to the extreme in its application to Wiedemann's results on torsion and set above quoted, we. should find that $\bar{\theta}=2400$, and that the couple necessary for zero resilience was fully double the maximum couple employed by Wiedemann (that corresponding to $\theta=1725$ ).

Various deductions might be drawn from the theory in connexion with the observed values of the constants in the empirical equations. I do not think that such deductions would be of any value except in connexion with a much wider experimental basis than that furnished above. I hope soon to be able to communicate the results of further observations.

IV. On the Mechanism of Electrical Conduction.-Part I. Conduction in Metals. By Charles V. Burton, D.Sc.*

1. THE view of electrical conduction which it is here my 1 object to explain receives general support from more than one consideration; for it leads to the conclusion that deviations from Ohm's Law must be quite inappreciable in the case of metallic conductors, and it goes far to explain, I think, why metals are so much less opaque than thei ${ }_{t}$ ordinary conductivities would lead us to infer. But it is no alone on such considerations that wo have to rely, for, as $i^{t}$ seems to me, the main conclusions are capable of exact demonstration ; and accordingly it would appear most convenient to commence with a few simple theorems, seeking afterwards to account for known phenomena by means of our definite results.

\section{Theorem I.}

In a region containing matter, there may be (and probably always are) some parts which are perfect insulators and some parts which are perfect conductors; but there can be no parts whose conductivity is finite-unless every finitely conductive portion is enclosed by a perfectly conductive envelope.

Before proceeding to the proof of this theorem, it may be remarked that the presence of the last clause in no way modities any application of our result, since the space within a perfectly conductive envelope is completely shielded from

* Communicated by the Physical Society : read April 13, 1894. 
the influence of external charges, currents, or magnets. In the present state of science, indeed, such words appear necessary to the completeness of demonstration, but they do not need to be considored in any of our deductions from the theorem, and for my own part I am persuaded that in reality there is nothing corresponding to the possibility which they suggest.

Consider now the case of any body whatever, at any temperature other than absolute zero. We know that electromagnetic radiations will spread out into the ether surrounding the body, and we mast suppose that the intermolecular spaces within the body are also traversed by electromagnetic disturbances. Let us suppose then, for a moment, that in the molecules of the body there are some finitely conductive portions which are not enclosed in perfectly conductive envelopes. The electromagnetic disturbances will give rise to currents of conduction in these portions, and accordingly energy will be degraded into a form which is not heat, since it consists, not in the motion or relative positions of molecules or appreciable parts of molecules, or in electromagnetic disturbances of the intervening ether, but in something much more fine-grained. We shall thus have a continual degradation of heat into energy of a lower form ; for the electromagnetic "damping" of the finitely conductive bodies involves a continual drain on the energy of internal radiation, and hence indirectly on the energy of the molecules, so that heat will be automatically dissipated in the interior of the body. This process, in which the radiative molecules are continually imparting to the ether more energy than they receive in return, may be compared to the surface conling of an isolated body which radiates towards colder surroundings.

Even if we suppose the finitely conductive bodies to be extremely small and their conductivity to be either extremely small or extremely great, it is not hard to see that the rate of absorption of heat must be tremendous; and when we consider (for example) the effect which even a very slow absorption, continued for millions of years, would have had on the temperature of our planet, we must admit that the absence of that dissipation of heat implied in the denial of Theorem I. has been established with an exactitude almost unparalleled. Thus the theorem is established.

3. In connexion with this result we are reminded that Poisson's theory of dielectrics requires the molecnles of insulating substances to possess some conductive portions, though whether the conductivity of such portions is finite or infinite 
is of no moment in electrostatics. On the other hand, both Ampère's theory of magnetism and Weber's theory of diamagnetism suppose the existence of perfectly conductive particles, and are thus strongly supported by our result.

In discussing Weber's theory of diamagnetism, Maxwell* points out that the currents excited in a perfectly conductive body by any external cause are entirely confined to the surface of the body. Thus the perfectly conductive bodies in Theorem I. may be replaced by perfectly conductive surfaces, without altering any of our conclusions; but it would be hard to decide whether a perfectly conductive geometrical surface is or is not a physical possibility without knowing more of electromagnetism-not to speak of ordinary matter.

\section{Theorem II.}

In metals, and in other non-electrolytes whose conductivity is finite, the transmission of currents must be effected by the intermittent contact of perfectly conductive particles.

For if there were not these intermittent contacts, any given two of the conductive particles would be either permanently in contact with one another, or permanently out of contact, and there would be only two cases to consider. If throughout the substance there extended continuous chains of (perfectly) conductive particles in contact with one another, the substance as a whole would be a perfect conductor; while in the absence of such chains of particles, the substance would be a perfect non-conductor. Finite conductivity can only exist when the contacts are intermittent.

\section{An immediate corollary is}

\section{THeorem III.}

If we suppose that in a substance at the absolute zero of temperature there is no relative motion amongst the molecules or amongst their appreciable parts, it follows that every substance at this temperature must have either infinite specific resistance (which need not imply infinite dielectric strength), or infinite conductivity.

For the denial of relative motion involves the denial of that intermittence of contact which in Theorem II. was shown to be necessary to finite conductivity.

This conclusion is in accordance with the experiments of

* 'Electricity and Magnetism,' 2nd ed. vol, ii. \$840. 
Dewar and Fleming* on the resistance of pure unalloyed metals at very low temperatures. In the case of all the pure metals examined by these anthors (platinum, gold, palladium, silver, copper, aluminium, iron, nickel, tin, magnesium, zinc, cadmium, lead, and thallium), the temperature-resistance curves are almost straight lines, and these, being produced, would pass very nearly through the point whose coordinates are zero temperature and zero resistance.

The same was not found to hold good for the temperatureresistance curves for alloys; but if these curves conld be pursued far enough by experiment, they must be found, I think, to terminate at the origin of coordinates, like those of the pure metals.

\section{Dissipation of Energi in a Conductor conveying a Current.}

In fig. 1 let $A$ and $B$ be two perfectly conductive particles (whether molecules or parts of the same or of different molecules we need not consider), and let them be approaching one another. Suppose also that there is an applied E.M.F. acting from right to left (as indicated by the large arrow). Then, generally speaking, A will be negatively electrified, owing to a provious encounter with some particle farther to the left, and for a similar reason $B$ will in general be positively electrified. When $A$ and $B$ collide, the usual effect is to leave $A$ on the whole positively electrified, and B negatively electrified.

Remembering that the conductivity of $A$ and $B$ is perfeet, let us consider what transformations of energy are effected by movements and collisions of this kind. Before the collision, A being negatively electrified is urged towards the right by the applied E.M.F., while B being positively electrified is urged towards the left: that is, $A$ and $B$ are urged together, and are gaining kinetic energy at the expense of the source of applied E.M.F. After the collision, the electrifications are, generally speaking, reversed, so that $\mathrm{A}$ and $\mathrm{B}$ are now being urged apart by the applied E.M.F., and continue to gain kinetic energy as before. Further, when particles such as $A$ and $B$ come into collision, so as to cause a readjustment of their electrifications, and also when they are in motion between two collisions, electromagnetic disturbances will be produced in the intermolecular ether; but since all

$$
\text { * Phil. Mag. Sept. 1893, p. } 271 .
$$


the conductive particles are perfectly conductive, no electromagnetic energy can penetrate within them. Thus the energy expended by the source of E.M.F. which maintains a steady current through a conductor is converted partly into additional energy of the molecules, and partly into electromagnetic disturbances of the intervening ether: that is, the dissipated energy takes the form of heat, as we know from experiment.

\section{OHM's LAW.}

In the case of a metal wire (especially one at a bright red heat), Ohm's Law has been verified with great exactitude, the results of the experiments designed by Maxwell and carried out by Chrystal being summed up by the latter in the following words *:-_" If we have a conductor [of iron, platinum, or German silver] whose section is a square centimetre, and whose resistance for infinitely small currents is an ohm, its resistance (provided the temperature is kept the same) is not diminished by so much as the $1 / 10^{\mathrm{x} 2}$ part when a current of a farad per second passes through it."

Now when a current is conveyed through a substance by intermittent contacts amongst a number of perfectly conductive particles, the effective conductivity depends firstly on the properties of the intermolecular medium, and secondly on the size, form, distribution, and movements of the particles themselves. In order that the resistance of the conductor may be sensibly constant-in order, that is, that the current transmitted may be sensibly proportional to the impressed E.M.F.- - two conditions must evidently be satisfied:-

(i.) For such values of impressed electromotive intensity as exist in the intermolecular spaces (say about .003 volt per $\mathrm{cm}$.) the relation between electromotive intensity and electric displacement must be sensibly linear.

(ii.) The forces which the particles of the substance experience owing to the impressed E.M.F. must be very small in comparison with the ordinary intermolecular forces, so that during the time of a single molecular excursion the motion of no particle is appreciably influenced by the presence of the E.M.F. If we suppose that in the conducting substance we can maintain a steady distribution of temperature which is independent of the current flowing through, this second condition implies that the particles of the substance under the steady distribution may be regarded as a system of perfect conductors, whose coordinates are explicitly given functions of the time, and are sensibly unalterable by an E.M.E.

* B. A. Report, 1876, p. 61 of Reports. 
impressed upon the system from without. This condition, combined with (i.), will evidently give us Ohm's Law.

Now the forces actually present and tending to modify the heat-movements are of two kinds : electromagnetie and electrostatic.

(a) Electromagnetic Forces.-The passage of a current through a conductor gives rise to a magnetic field, whieh may or may not appreciably affect the conductivity. The thin iron wire used by Prof: Chrystal was $0021 \mathrm{~cm}$. in radius, and the greatest value of the magnetic force due to a current of 1 ampere per square centimetre of cross section would be in absolute measure about 0013 (at the surface of the wire), the square of the greatest magnetic force being thus about 0000017 . The average value of (magnetic force $)^{2}$ over the cross section of the wire would be half of this, or $\cdot 00000085$; that is, about $\cdot 0000039$ of the square of the terrestrial "total force" in these parts.

Now Lord Kelvin found * that the change of resistance due to transverse magnetization of an iron plate by a powerful Ruhmkorff electromagnet was only just decided enough to be distinctly appreciated with the apparatus which he employed, and we may therefore conclude that in Prof. Chrystal's iron wire no perceptible change of resistance could have been produced by the magnetic field of the current. In other metals the effect must be still more insigniificant.

On the other hand, the longitudinal magnetization of an iron wire perceptibly increases its electrical resistance, so that it would be easy to construct a simple conductor whose resistance at a given temperature was a function of the current-strength. For let a flat bobbin be wound with iron wire, so that each turn has the form of an elongated rectangle, and then let a further quantity of iron wire be wound in a similar circuit embracing the first. Finally let the coils be joined in series with a source of E.M.F. When a current is sent through the circuit, each coil will magnetize longitudinally some parts of the wire of the other coil, and so, for a given temperature of the wire, the resistance will increase with the current.

(b) Electrostatic Forces.-Let us attempt to calculate the electrostatic energy per cubic centimetre which a mass of iron possesses in virtue of a current flowing through it with a "density" "of 1 ampere per cm. ${ }^{2}$ To do this we must assume some value for the specific inductive eapacity of iron $\dagger$, and

* Phil. Trans. 1856, especially pp. 747-749.

† In electrostatic measurements conductors appear to have an infinite specific inductive capacity; but here, where the potential really varies from point to point through the metal, it is the true (finite) specific inductive capacity which cuncerns us. 
in order to take a sufficiently unfavourable view of the question, let us assume the value to be as high as 20. Taking the specific resistance of iron in electromagnetic measure to be 10,000 , and remembering that 1 ampere $=\cdot 1$ absolute unit, we have for the electromotive intensity 1000 electromagnetic units of potential per cm., i.e. $1000 \div\left(3 \times 10^{10}\right)$ electrostatic units per $\mathrm{cm}$. Hence the electrostatic energy per c. c. due to the impressed E.M.F.

$$
=\frac{20}{8 \pi\left(3 \times 10^{7}\right)^{2}} \text { ergs ; }
$$

while to calculate the thermal energy per c. c. at " a bright red heat"- - the temperature of the iron in the British Association experiments-we have:-

Temperature above absolute zero (say) $=727+273$

Density of iron . . . . . . . $=7 \cdot 8$,

Specific heat. . . . . . . $=113$,

One gram-water-degree of heat $\quad=42 \times 10^{6}$ ergs.

Thus (roughly speaking) the thermal energy per c. c. reckoned from absolute zero

$$
=1000 \times 7 \cdot 8 \times 113 \times 42 \times 10^{6} \text { ergs. }
$$

A comparison of these results gives

$$
\frac{\text { electrostatic energy due to impressed E.M.F. }}{\text { thermal energy }}=\frac{1}{4 \times 10^{22}}
$$

only, even on our assumption that the specific inductive capacity of iron in electrostatic measure is as high as 20 . If we suppose that half the thermal energy is potential and half kinetic, then the electrostatic energy would be $1 \div\left(2 \times 10^{22}\right)$ of the thermal kinetic energy; that is, would be equal to the additional energy required to increase the existing velocity of every particle by one part in $2 \times 10^{22}$. When due account is taken of these results it is not surprising to find that in iron at a given temperature the specific resistance for a currentdensity of one ampere per $\mathrm{cm}^{2}$ differs from the specific resistance for an infinitesimal current-density by less than one part in $10^{12}$.

The same remarks apply with even greater force to platinum and German silver, the other metals examined by Prof. Chrystal, since the magnetic influence of the current on the resistances of these metals must be far less than even in the case of iron.

From considerations similar to these, we should expect in all true conducting substances (even in those having marked 
magnetic properties) to find a sensibly linear relation connecting eurrent-density with electromotive intensity in the neighbourhood of each point.

\section{Contaot E.M.F. and the Peltier Effect.}

It will now appear that, by assuming in each molecule a mere arrangement of conducting and non-conducting parts, we may realize what is at all events a working model of contact E.M.F. and of the Peltier effect; and even should our model serve no other purpose, it directs our attention to a possibility which seems so far to have been overlooked, and which must, indeed, continue to be overlooked so long as each metal is regarded as homogeneous. As it not my object to state with becoming vagueness an hypothesis as to the nature of Peltier's phenomenon, but rather to picture as clearly as possible a mechanism whose principle may perhaps be suggestive of the truth, 1 shall assume for the molecules such a structure and distribution as appear most favourable to simplicity of treatment.

Suppose, then, that in one of the metals with which we have to deal, each molecule is of the form indicated in fig. 2 : a central perfectly conductive body, charged (say) positively, being completely surrounded by an insulating layer, and this again being partially (though not completely) enclosed by a number of perfectly conductive particles. It will be convenient to suppose that in each molecule these outer particles are all electrically connected with one another. When two such molecules come into collision, the outer particles of the one may not in general be reduced to the same potential as those of the other, for the electrical oscillations occasioned by the contact may not have time to subside into insignificance before the encounter is ended. But the general tendency of a collision between two molecules will be towards an equalization of the potentials of the outer particles, and the average values of potentials and charges amongst any considerable number of molecules will be the same as if, during each molecular encounter, this equalization has been completely effected.

It will here be convenient to introduce the term "conduction-potential," and as we proceed the following definitions will be found useful:-

The conduction-potential of a molecule is the potential (or average potential) of its outer conductive particles.

The conduction-potential at a point within a metallic body is the average conduction-potential of the molecules in the neighbourhood of that point. 
Let us now turn to the case of a considerable mass of metal, made up entirely of such molecules as that sketched in fig. 2; every molecule having the same structure and the same internal charge. Let us suppose also that the mass of metal is at the same temperature and in the same physical condition throughout, so that the average distance between adjacent molecules is the same in the neighbourhood of each point. If the metallic body is subjected to electrostatic induction, it is easy to see that no electric influence from without can penetrate far beyond the surface; for the outer particles of the superficial molecules, owing to their intermittent contacts with one another, will screen the interior of the metal as a network of continuous wires might do, and at a depth of a rery few molecules beneath the surface the screening will be practically complete. Thus any charge communicated to the conductor will be confined entirely to the superficial layers of molecules; and it follows that if between any two points well within the metal there were a difference of average conduction-potential, there would be a general flow of electrification from the higher towards the lower potential. The average conduction-potential within the metal does not therefore vary from point to point, and account being taken of the equality of the internal charges of the molecules, it follows that the average potential of the intermolecular ether is similarly free from variation. This implies that the total electrification of any considerable assemblage of molecules within the metal is sensibly zero; so that the average charge on the outer conductive particles of each molecule is equal and opposite to the fixed charge on the central particle. The conclusions of this paragraph have been necessarily confined to molecules not too near the surface; for although the general tendency of an encounter between two molecules is always to equalize their conductionpotentials, these potentials change somewhat after the molecules have become separated, and near the free surface of the body the change will be systematically greater for the molecule which moves outwards after collision than for that which moves inwards.

We may now try to realize what will happen when contact takes place between two metals whose molecules are constructed on the general plan of fig. 2, while those of the one metal are not identical with those of the other. The most simple and intelligible view will be obtained by supposing the molecule of the one metal to have a positively charged central particle, while the molecule of the other has its central particle negatively charged (fig. 3). Consider what would 
occur if we could start from a condition in which each of the border molecules, A, B, C, D, \&c., had on the whole no charge ; a condition, that is, in which the outer particles of each molecule had a charge equal and opposite to the fixed charge

Fig. 3.
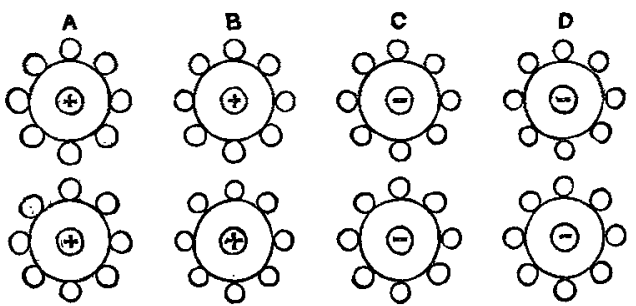

of the central particle. (We have already seen that this is true for the average molecule within a homogeneous metallic mass.) Now when B and C come into collision, it is evident that the outer particles of $B$ will lose some of their negative electrification, while the outer particles of $\mathrm{C}$ will lose some of their positive; and thus we see that when the distribution of conduction-potential has become steady, such border particles as $\mathrm{B}$ or $\mathrm{C}$ will have on their outer particles a (negative or positive) charge less than the (positive or negative) fixed central charge, and to a smaller extent the same will be true of particles (such as $\mathrm{A}, \mathrm{D}$, \&c.) more remote from the border. But when the settled condition has been reached, the passage (say) of $B$ backwards and forwards between $A$ and $C$ will not change the distribution of charges amongst the molecules; and the condition that no change of the kind shall take place is that when two molecules are in a position to collide their conduction-potentials shall be equal. Now when B approaches C (which has on the whole a negative charge) its potential is lowered, and when $B$ returns towards $A$ its potential rises again, so that if $\mathrm{B}$ in its backward and forward motion is not to act as a systematic carrier of electrification between $\mathrm{A}$ and $\mathrm{C}$, the conduction-potential of $\mathrm{A}$ must be higher than that of $\mathrm{C}$. Similarly, by considering $\mathrm{C}$ as moving backwards and forwards between $B$ and $D$, we can see that $B$ must have a higher conduction-potential than $\mathrm{D}$.

This gives us a contact-difference of conduction-potential.

If we suppose our analysis to become a trifle less penetrating, such molecules as B (and to a less extent A, \&c.) will appear to be positively electrified, C, D, \&c. will appear to be negatively electrified, and molecules farther removed from the border will appear unelectrified. With still less micro- 
scopical vision, we shall find the conduction-potential constant from point to point throughout the mass of metal on either side of the surface of separation; but as we approach that surface from the left, the conduction-potential begins to diminish, changing very rapidly by a finite amount as we pass through the boundary.

We now come to a theorem which is certainly true for our model, and which seems to me as certainly true for any mechanism which could be devised to represent the Peltier effect; but for the sake of avoiding questions of too controversial a character, the statement may be made in this conditional form:

\section{Theorem IV.}

In our model, the contact-difference of conduction-potential between two metals is equal to the coefficient of the Peltier effect. For when a molecule at the junction is moving backwards and forwards between places of different potentials, provided no current flows through the junction, as much electrification is carried from the lower to the higher potential as from the higher to the lower, and on the whole there is no transformation of electric energy into heat, or vice vers $\hat{x}$. But when a current flows across the junction from the metal of lower to that of higher conduction-potential, the molecules at the junction are persistently carrying more electrification from the lower to the higher potential than they bring back with them on their return, and thus on the whole the movements of the molecules at the junction are systematically opposed by electrostatic forces. It is evident from elementary considerations that, the quantity of electricity which has crossed the junction, multiplied by the step of (conduction-) potential up which it has passed, is the measure of the total work done by the molecules against electrostatic forces, and is therefore the measure of the heat absorbed. Similarly, when a current has been flowing from the metal of higher to that of lower conduction-potential, the quantity of electricity which has crossed the junction, multiplied by the (negative) step of conduction-potential, is the measure of the (negative) heat absorbed; that is, numerically, of the heat given out. Hence, in our model, the coefficient of the Peltier effect is equal to the contact-difference of conduction-potential.

Again, generally speaking, we may expect a difference of conduction-potential between a hotter and a colder portion of the same metal, owing to the increase of molecular distances which rise of temperature produces; and it is evident that (on our model) the specific heat of electricity for any metal is equal to the rise of conduction-potential for one degree rise of temperature.

Phil. Mag. S. 5. Tol. 38. No. 230. July 1894. $\quad$ F 
9. From these results we pass on to

Theorem V.

For any pair of metals at the absolute zero of temperature, the Peltier effect vanishes.

This is evidently true for our model, for when the molecules are all reduced to relative rest, and there is permanent instead of intermittent contact amongst their outer particles, the conduction-potential will be uniform throughout both metals, and at the junction there will be no Peltier effect. But whatever view we take of the nature of the phenomenon, the proposition is necessarily true. For if the Peltier effect had a finite value for a pair of metals at the absolute zero of temperature, we could cause an absorption of heat by sending a current through the junction in the proper direction; and this is impossible, since there is no heat to be absorbed.

\section{Volta E.M.F.'s.}

We must now consider a possibility suggested by our model, and referred to in the opening sentence of $\$ 8$. It is not difficult to see that, with molecules constructed on the plan of fig. 2, even when all measurements are made in vacuo, the conduction-potential of a mass of metal is not in general the same as the potential estimated by work done on an external charged body, or by electrification induced on a second mass of metal, insulated from the first,-potential measured in the latter way being called for distinction the induction-potential.

We may realize this most easily by considering the case of two metals in contact at the absolute zero of temperature, for then, in accordance with the last section, the Peltier effect at the junction vanishes, and the conduction-potential is the same throughout both metals; while on the other hand the difference of induitionpotential may be finite. 'Liet fig. 4 represent diagrammatically a very large number of molecules which are at rest with their outer conductive particles in electrical contact throughout. Let the fixed central charge of each molecule be positive. Then, if the outer conductive particles of each molecule formed a Fig. 4.

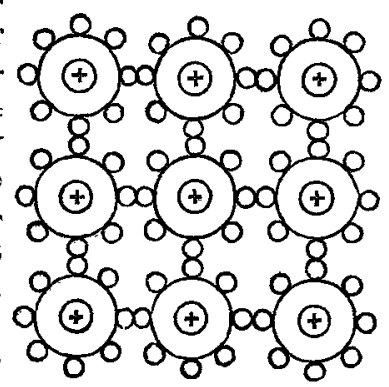
complete envelope around the central charge, the inductionpotential of the metal would be identical with its conductionpotential, and the same as if the fixed central charges did 
not exist. But since we suppose the fixed charge in each molecule to be incompletely screened by the outer particles, it follows that at external points in the immediate neighbourhood of the metallic body the potential is raised above the conduction-potential by the fixed central charges. If these last were negative instead of positive, the potential just outside the metallic mass would be lower than the conduction-potential; and we may suppose that at any given temperature (such as the absolute zero with which we are dealing) the difference between the conductionpotential of a metallic body and the potential just outside the body depends upon the nature of the metal. Thus, even in racuo, if two metals at the absolute zero of temperature be connected together so as to have the same conductionpotential, their induction-potentials may be different; and in general, whatever the temperature of the metals in contact, we may expect an inequality between difference of conductionpotential and the difference of induction-potential.

Before attempting to devise a model of Peltier's phenomenon and of electromotive forces of contact, I had held the opinion-in common, I believe, with the majority of disputants in the contact-force controversy - that the inductive measurement of potential-differences in a sufficiently perfect vacuum must conclusively decide the points at issue. But if in reality there should be, as the model suggests, a difference between conduction-potentials and induction-potentials, we must not rely upon inductive experiments, even in a perfect vacuum, to determine the seats of electromotive force in a voltaic cell. For when we are dealing with the flow of currents through metals, it is the conduction-potential which concerns us.

\section{The Transparency of Metals.}

A difficulty in connexion with this subject is stated by Maxwell in the following well-known passage * :- "Gold, silver, and platinum are good conductors, and yet, when formed into very thin plates, they allow light to pass through them. From experiments which I have made on a piece of gold-leaf, the resistance of which was determined by Mr. Hockin, it appears that its transparency is very much greater than is consistent with our theory, unless we suppose that there is less loss of energy when the electromotive forces are reversed for every semi-vibration of light than when they act

* 'Electricity and Magnetism,' 2nd ed. vol. ii. \$800. Wien (Wiedemann's Annalen, xxxv. pp. 41-62) found a silver film to have only such an opacity as would be deduced from about $1 / 440$ of its actual conductivity. 
for sensible times, as in our ordinary experiments." Now we have seen that conduction is not a perfectly continuous phenomenon, but is due to innumerable encounters among perfectly conductive particles, and without entering upon any ealculations (which indeed would be a difficult matter) we can see that there are, broadly speaking, two reasons why the opacity of metals is so much smaller than is indicated by Maxwell's analysis : these are, heterogeneity of structure and intermittence of contact.

To realize the influence of heterogeneity of structure without the complication of intermittent contacts, take the case of a metal at the absolute zero of temperature. We have then virtually to deal with a network of perfect conductors, constituting a body which as a whole has perfect conductivity, and of which even an excessively thin film would be an effectual barrier to electromagnetic waves, provided that the wavelength were great enough to justify us in treating the metal as homogeneous. But if we consider an extreme case, where the wave-length of the disturbance is negligible in comparison with the dimensions of a single conductive particle, a very thin layer of the metal will be far from absolutely opaque. For the conditions of the problem will then be the same as if we had ordinary luminous radiations obstructed by an agglomeration of perfectly reflecting bodies of appreciable size. Of course these extreme conditions are not realized in the case of the light transmitted by a metaliic film; but if we may suppose that the diameter of a conductive particle is not quite negligible in comparison with a wave-length of light, it is clearly to be expected that very thin layers of the metal will fall short of that absolute opacity which in this case would follow from the assumption of homogeneity.

When we pass to the consideration of metals at ordinary temperatures, the conductivity for steady currents is finite; but for electromagnetic waves of short period we cannot even treat the metal as an agglomeration of finitely conductive particles continuously in contact with one another. It is evident that the shorter we make the period of the electromagnetic disturbance in comparison with the average intercollisionary period of a (perfectly) conductive particle, the more nearly do the particles act as if permanently insulated from one another, and the less efficiently does the metal perform the functions of an electromagnetic screen.

Further considerations might be added concerning the average interchange of electrification between colliding particles when the electromotive intensity tending to produce such interchange is very rapidly alternating; but enough has 
been said to show that the opacity of conductors must be far less for luminous radiations than for electromagnetic disturbances of long period, and we may fairly expect, I think, that the transparency of metals is to be explained without attributing any new properties to the electromagnetic field.

The second part of this paper will deal with electrolytic conduction and disruptive discharge.

\section{Note added April 30th.}

In the course of the discussion Prof. S. P. Thompson objected to the arrangement of molecules in rectangular order, and he further suggested that the arguments might only be applicable in two dimensions. I had omitted to mention that the figures were intended to be sectional views of threedimensional models, while the rectangular arrangement of molecules was merely adopted to save prolixity in the descriptions, and was so far from being essential to the investigation that the case of irregularly distributed coordinates and velocities was constantly before my mind. Another point raised by Prof. Thompson must also be considered here : in $\$ 6$ it does not necessarily follow that two conductive particles oppositely charged like A and B (fig. 1), approaching one another and subject to the influence of an external E.M.F. acting from right to left, would have the signs of their respective electrifications reversed by a momentary contact; in some encounters the readjustment of electrifications might even be in the opposite sense; but I think we may safely admit that in the long run the effect of innumerable collisions amongst such conducting particles as $\mathrm{A}$ and $\mathrm{B}$ will be to transfer electrification in the direction of the impressed E.M.F.

Prof. Rücker recalled a difficulty, which Lord Kelvin pointed out some time ago, in connexion with the collisions between molecules. If wo suppose the molecules to be constituted like little pieces of elastic solid, every collision will cause some additional amount of translational energy to be converted into energy of vibration, and heat-energy will be continually running down into energy of shriller and shriller vibrations, that is, into energy of a lower form. In the foregoing pages, electrical contact between particles is supposed to oceur during a collision, and Prof. Rücker remarked that the method suggested for avoiding an electromagnetic degradation of energy left untouched the corresponding mechanical difficulty. I have made some attempt to deal with this 
mechanical question in a previous paper*, where it was shown $(\$ \$ 10,11)$ that, granted the fundamental assumption and an infinite propagation-velocity for gravitational stress, we may construct an atom having a finite number of freedoms. But in whatever way mechanical degradation of energy were eliminated, the difficulty of electromagnetic degradation would also have to be met, and without the doctrine laid down in Theorem I. there appeared to me to be no means of escape. Without making any assumption as to the constitution of a molecule or the nature of a collision, we may admit that in any body not absolutely cold there are particles in relative motion, so that two neighbouring particles are sometimes nearer together and sometimes farther apart. To realize the intermittence of contact required by Theorem II., we have only to suppose that when (but not until) the proximity of two particles has reached a certain limit electritication is capable of passing freely from one to the other.

The question of perfect or imperfect conductivity in the ultimate particles of bodies must be of importance in relation to the constitution of matter and its connexion with the ether ; and whether or not the demonstrations above can be generally accepted as conclusive, the subject is certainly one which will repay further investigation.

\section{Some Observations on Diffraction. $B y$ W. B. Сroft, $M . A . \dagger$}

[Plates I.-IV.]

$I^{1}$ $T$ is proposed to illustrate various forms of this phenomenon by photographs $\ddagger$ produced directly from the waveinterference.

After the inauguration of the idea about 1665 by Grimaldi, Hooke, and Huygens, there was little progress, either in extended observation or in philosophical grasp of the principles, until the beginning of this century. Since that time the subject has been treated in two ways.

1st. The Diffraction of Fraunhofer and Schwerd.

This kind is familiar to many through the observations of Sir John Herschel of Diffraction in a Telescope. It is sometimes described as the Diffraction from Parallel Light.

* "A Theory concerning the Constitution of Matter," Phil. Mag. February 1892, p. 191.

+ Communicated by the Physical Society: read January 26, 1894.

I It is not convenient to reproduce all the photographs: the selected figures $2,3,4,10,12,13,71,72,75,83$ will be found on Plates $I_{\text {.-IV. }}$. 